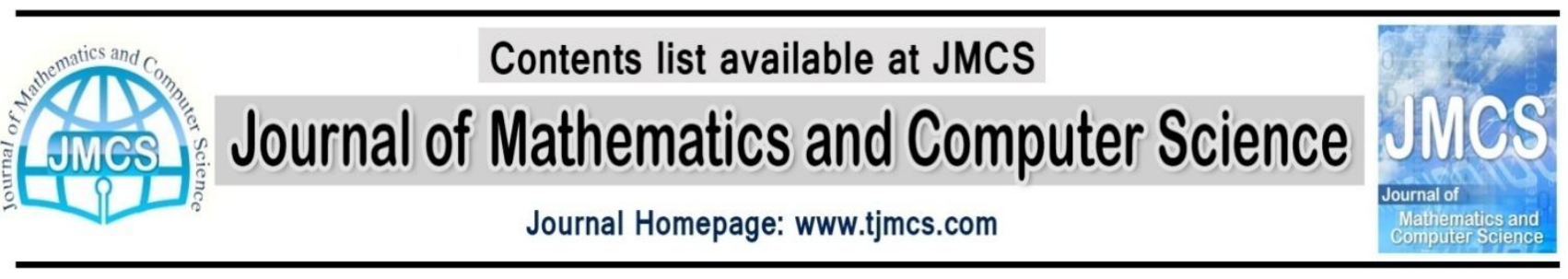

\title{
AN ITERATIVE METHOD FOR SEMIGROUPS OF NONEXPANSIVE MAPPINGS
}

\author{
A. Dianatifar, F. Golkar, A. M. Forouzanfar \\ Department of Mathematics, Shahid Chamran University of Ahvaz, Ahvaz, Iran \\ E-mail \\ Dianati.math@gmail.com \\ f-golkar@phdstu.scu.ac.ir \\ am.forouzanfar@scu.ac.ir
}

Article history:

Received May 2014

Accepted June 2014

Available online July 2014

\begin{abstract}
We introduce an iterative method for finding a common fixed point of a semigroup of infinite family of nonexpansive mappings in Hilbert space, with respect to a sequence of left regular means defined on an appropriate space of bounded real valued functions of the semigroup. we prove the strong convergence of the proposed iterative algorithm to the unique solution of a variational inequality, which is the optimality condition for a minimization problem.
\end{abstract}

Keywords: Hilbert space, Amenable semigroups, Common fixed point, Nonexpansive mappings.

\section{Introduction}

Let $\mathrm{H}$ be a real Hilbert space. Assume A is strongly positive, that is there is a constant $\bar{\gamma}$ with the property:

$$
\langle A x, x\rangle \geq \bar{\gamma}\|x\|^{2} .
$$


Let $\left\{T_{i}\right\}_{i=1}^{\infty}$ be a sequence of nonexpansive mappings of $\mathrm{H}$ into itself, we shall assume that $F:=\bigcap_{i=1}^{\infty} F i x T_{i} \neq \varnothing$, and let $\left\{\lambda_{i}\right\}_{i=1}^{\infty}$ be a sequence of nonnegative real numbers in $[0,1]$. for $n \geq 1$, define a mapping $W_{n}$ of $\mathrm{H}$ into itself as follows:

$U_{n, n+1}=I$

$U_{n, n}=\lambda_{n} T_{n} U_{n, n+1}+\left(1-\lambda_{n}\right) I$

$U_{n, 2}=\lambda_{2} T_{2} U_{n, 3}+\left(1-\lambda_{2}\right) I$

$W_{n}:=U_{n, 1}=\lambda_{1} T_{1} U_{n, 2}+\left(1-\lambda_{1}\right) I$

Y.Yao in [12] introduced an iterative algorithm to appropriate the common fixed points of an infinite family of nonexpansive self mapping in a real Hilbert space as follows:

Let $x_{0} \in H$ is arbitrarily chosen and

$$
x_{n+1}=\alpha_{n} \gamma f\left(x_{n}\right)+\beta_{n} x_{n}+\left(\left(1-\beta_{n}\right) I-\alpha_{n} A\right) W_{n} x_{n}
$$

Where $W_{n}$ is a sequence defined by (1.1), $\mathrm{f}$ be a contraction on $\mathrm{H}$ with

coeffecient $\bar{\gamma}>0$ such that $0<\gamma<\frac{\bar{\gamma}}{\alpha}$

Under the assumption that sequences $\left\{\alpha_{n}\right\},\left\{\beta_{n}\right\}$ satisfy the following conditions:

$\left(C_{1}\right): \lim _{n \rightarrow \infty} \alpha_{n}=0$

$\left(C_{2}\right): \sum_{n=1}^{\infty} \alpha_{n}=\infty$

$\left(C_{3}\right): 0<\liminf \beta_{n}<\limsup \beta_{n}<1$,

Yao proved that the sequence $\left\{x_{n}\right\}$ converges strongly to the unique solution of the variational inequality

$$
\left\langle(A-\gamma f) x^{*}, x-x^{*}\right\rangle \geq 0 \quad x \in F .
$$

In this paper motivated and inspired by Yao, we introduce a composite iteration schem as follows:

$$
x_{n+1}=\alpha_{n} \gamma f\left(x_{n}\right)+\beta_{n} x_{n}+\left(\left(1-\beta_{n}\right) I-\alpha_{n} A\right) T_{\mu_{n}} W_{n} x_{n}
$$


Let $\mathrm{S}$ be a semigroup, $\varphi=\left\{T_{t}: t \in S\right\}$ is a nonexpansive semigroup on $\mathrm{H}$ such that Fix $\varphi=\bigcap_{t \in S} F i x T_{t} \neq \varnothing, \mathrm{X}$ is a left invariant subspace of the space of all bounded real valued functions defined on $\mathrm{S}$ such that $1 \in X$, the mapping $t \rightarrow\left\langle T_{t}(x), y\right\rangle$ is an element of $\mathrm{X}$ for each $x, y \in H$ and $\left\{\mu_{n}\right\}$ is a sequence of means on X. Our purpose here is to introduce the general iterative algorithm for approximating the common fixed points of left amenable semigroup of nonexpansive mapping and infinite family of nonexpansive mappings which also solve some variational inequalities.

\section{PRELIMINARIES}

Let $\mathrm{S}$ be a semigroup and let $\mathrm{B}(\mathrm{S})$ be the space of all bounded real valued functions defined on $\mathrm{S}$ with the supremum norm. For $s \in S$ and $f \in B(S)$ we write $l_{s}$ and $r_{s}$ on $\mathrm{B}(\mathrm{S})$ by $\left(l_{s} f\right)(t)=f(s t)$ and $\left(r_{s} f\right)(t)=f(t s)$ for each $t \in S$ and $f \in B(S)$. Let $\mathrm{X}$ be a subspace of $\mathrm{B}(\mathrm{S})$ containing 1 and $X^{*}$ be its topological dual. An element $\mu$ of $X^{*}$ is said to be a mean on $\mathrm{X}$ if $\|\mu\|=\mu(1)=1$. We often write $\mu_{t}(f(t))$ instead of $\mu(f)$ for $\mu \in X^{*}$ and $f \in X$. Let $X$ be left invariant ( resp. right invariant), i.e $l_{s}(X) \subset X$ (resp. $r_{s}(X) \subset X$ ) for each $s \in S$. A mean $\mu$ on $\mathrm{X}$ is said to be left invariant (resp. right invariant) if $\mu\left(l_{s} f\right)=\mu(f)$ (res $\mu\left(r_{s} f\right)=\mu(f)$ for each $s \in S$ and $f \in X$. X is said to be left (resp. right) amenable if $\mathrm{X}$ has a left (resp. right) invariant mean. $\mathrm{X}$ is amenable if $\mathrm{X}$ is both left and right amenable. As is well known, $\mathrm{B}(\mathrm{S})$ is amenable when $\mathrm{S}$ is a commutative semigroup or a solvable group. A net $\left\{\mu_{\alpha}\right\}$ of means on $\mathrm{X}$ is said to be strongly left regular if $\lim _{\alpha}\left\|l_{s}^{*} \mu_{\alpha}-\mu_{\alpha}\right\|=0$ for each $s \in S$ where $l_{s}^{*}$ is the adjoint operator of $l_{s}$.

Let $\mathrm{C}$ be a nonempty closed and convex subset of a reflexive Banach space $\mathrm{E}$. A family $\varphi=\left\{T_{t}: t \in S\right\}$ is called a nonexpansive semigroup on $\mathrm{C}$ if for each $t \in S$ the mapping $T_{t}: C \rightarrow C$ is nonexpansive and $T_{s t}=T_{s} o T_{t}$ for each $s, t \in S$. We denote by Fix $(\varphi)$ the set of common fixed points of $\varphi$.

Lemma 2.1. [1, 4] Let $\mathrm{f}$ be a function of semigroup $\mathrm{S}$ into a reflexive Banach space $\mathrm{E}$ such that the weak closure of $\{f(t): t \in S\}$ is weakly compact and let $\mathrm{X}$ be a subspace of $\mathrm{B}(\mathrm{S})$ containing all functions $t \rightarrow<f(t), x^{*}>$ with $x^{*} \in E^{*}$. Then, for any $\mu \in X^{*}$, there exists a unique element $f_{\mu}$ in E such that $\left\langle f_{\mu}, x^{*}>=\mu_{t}<f(t), x^{*}>\right.$, for all $x^{*} \in E^{*}$. Moreover, if $\mu$ is a mean on $\mathrm{X}$, then

$$
\int f(t) d \mu(t) \in \overline{c o}\{f(t): t \in S\}
$$

We can write $f_{\mu}$ by $\int f(t) d \mu(t)$.

Lemma 2.2. [1, 4] Let $\mathrm{C}$ be a nonempty closed convex subset of a Hilbert space 
$\mathrm{H}, \varphi=\left\{T_{t}: t \in S\right\}$ be semigroup from $\mathrm{C}$ into $\mathrm{C}$ such that $F(\varphi) \neq \varnothing$ and the mapping $t \rightarrow<T_{t}(x), y>$ be an element of $\mathrm{X}$ for each $x \in C$ and $y \in H$, and $\mu$ be a mean on $\mathrm{X}$. If we write $T_{\mu}(x)$, instead of $\int T_{t}(x) d \mu(t)$, then the followings hold:

(i) $T_{\mu}$ is nonexpansive mapping from $\mathrm{C}$ into $\mathrm{C}$.

(ii) $T_{\mu}(x)=x$, for each $x \in$ Fix $(\varphi)$.

(iii) $T_{\mu}(x) \in \overline{c o}\left\{T_{t}(x): t \in S\right\}$, for each $x \in C$.

(iv) If $\mu$ is left invariant, then $T_{\mu}$ is a nonexpansive retraction from $\mathrm{C}$ onto Fix $(\varphi)$.

Lemma 2.3. [3] Let $\mathrm{C}$ be a nonempty closed convex subset of $\mathrm{H}$ and $T: C \rightarrow C$ a nonexpansive mapping with $F(T) \neq \varnothing$. If $\left\{x_{n}\right\}$ is a sequence in $\mathrm{C}$ weakly converging to $x$ and if $\left\{(I-T) x_{n}\right\}$ converges strongly to $\mathrm{y}$, then $(I-T) x=y$.

Recall the metric (nearest point) projection $P_{C}$ from a Hilbert space $\mathrm{H}$ to a closed convex subset $\mathrm{C}$ of $\mathrm{H}$ is defined as follows: give $x \in H, P_{C}(x)$ is the only point in $\mathrm{C}$ with the property:

$$
\left\|x-P_{C}(x)\right\|=\inf \{\|x-y\|: y \in C\}
$$

It is well-known that $P_{C}$ is a nonexpansive mapping of $\mathrm{H}$ onto $\mathrm{C}$.

Lemma 2.4. [3] Let $\mathrm{C}$ be a nonempty convex subset of a Hilbert space $\mathrm{H}$ and $P_{C}$ be the metric projection mapping from $\mathrm{H}$ onto $\mathrm{C}$. Let $x \in H$ and $y \in C$ then, the followings are equivalent:

i) $y=P_{C}(x)$

ii) $<x-y, y-z>\geq 0 \quad \forall z \in C$.

Lemma 2.5. [9] Let $\left\{x_{n}\right\}$ and $\left\{y_{n}\right\}$ be bounded sequences in a Banach space $\mathrm{E}$ and let $\left\{\alpha_{n}\right\}$ be a sequence in $[0,1]$ with $0<\liminf _{n \rightarrow \infty} \alpha_{n} \leq \limsup _{n \rightarrow \infty} \alpha_{n}<1$. Suppose $x_{n+1}=\alpha_{n} x_{n}+\left(1-\alpha_{n}\right) y_{n}$, for all integers $n \geq 0$, and limsup $\left.\left\|y_{n+1}-y_{n}\right\|-\left\|x_{n+1}-x_{n}\right\|\right) \leq 0$.

Then, $\lim _{n \rightarrow \infty}\left\|y_{n}-x_{n}\right\|=0$.

The following lemma is an immediate consequence of the inner product on $\mathrm{H}$.

Lemma 2.6. For all $x, y \in H$, there holds the inequality

$$
\|x+y\|^{2} \leq\|x\|^{2}+2<y, x+y>\text {. }
$$

Lemma 2.7. [5] Let $\mathrm{C}$ be a nonempty closed convex subset of a Hilbert space $\mathrm{H},\left\{T_{i}: C \rightarrow C\right\}$ be an infinite family of nonexpansive mappings with $\bigcap_{i=1}^{\infty} F i x\left(T_{i}\right) \neq \varnothing,\left\{\lambda_{i}\right\}$ be a real sequence such that $0<\lambda_{i} \leq b<1, \forall i \geq 1$. Considering $W_{n}$ which is defined by (1.1) we have:

(1) $W_{n}$ is nonexpansive and Fix $\left(W_{n}\right)=\bigcap_{i=1}^{n} F i x\left(T_{i}\right)$ for each $n \geq 1$ 
(2) for each $x \in C$ and for each positive integer $\mathrm{j}, \lim _{n \rightarrow \infty} U_{n, j} x$ exists.

(3) The mapping $W: C \rightarrow C$, defined by

$$
W x:=\lim _{n \rightarrow \infty} W_{n} x=\lim _{n \rightarrow \infty} U_{n, 1} x, \forall x \in C .
$$

is a nonexpansive mapping satisfying $\operatorname{Fix}(W)=\bigcap_{i=1}^{\infty} \operatorname{Fix}\left(T_{i}\right)$ and is called the W-mapping generated by $T_{1}, T_{2}, \ldots$ and $\lambda_{1}, \lambda_{2}, \ldots$.

Lemma 2.8. [8] Let $\mathrm{C}$ be a nonempty closed convex subset of a Hilbert space $\mathrm{H},\left\{T_{i}: C \rightarrow C\right\}$ be a countable family of nonexpansive mappings with $\bigcap_{i=1}^{\infty} F i x\left(T_{i}\right) \neq \varnothing,\left\{\lambda_{i}\right\}$ be a real sequence such that $0<\lambda_{i} \leq b<1, \forall i \geq 1$. If $\mathrm{D}$ is any bounded subset of $\mathrm{C}$, then

$$
\limsup _{n \rightarrow \infty}\left\|x-W_{n} x\right\|=0 \text {. }
$$

Lemma 2.9. [3] Let $\left\{a_{n}\right\}$ be a sequence of nonnegative real numbers such that

$$
a_{n+1} \leq\left(1-b_{n}\right) a_{n}+b_{n} c_{n}, \quad \forall n \geq 0
$$

where $\left\{b_{n}\right\}$ and $\left\{c_{n}\right\}$ are sequences of real numbers satisfying the following conditions

(i) $\left\{b_{n}\right\} \subset[0,1], \sum_{n=0}^{\infty} b_{n}=\infty$

(ii) either $\limsup _{n \rightarrow \infty} c_{n} \leq 0$ or $\sum_{n=0}^{\infty} p_{n} c_{n} \mid<\infty$

Then $\lim _{n \rightarrow \infty} a_{n}=0$.

Lemma 2.10. [2] Assume that $\mathrm{A}$ is a strongly positive bounded operator on a Hilbert space $\mathrm{H}$ with the coefficient $\bar{\gamma}>0$ and $0<\rho<\|A\|^{-1}$.

Then, $\|I-\rho A\| \leq 1-\rho \bar{\gamma}>0$.

\section{Main results}

Theorem 3.1. Let $\mathrm{f}$ be a contraction on $\mathrm{H}$ with coefficient $0<\alpha<1$. Let $\mathrm{A}$ be a strongly positive operator on $\mathrm{H}$ with coefficient $\bar{\gamma}>0$. Let $\left\{\mu_{n}\right\}$ be a left regular sequence of means on $\mathrm{X}$ such that $\lim _{n \rightarrow \infty}\left\|\mu_{n+1}-\mu_{n}\right\|=0$. Let $\left\{T_{i}\right\}_{i=1}^{\infty}$ be a sequence of nonexpansive selfmapping of $\mathrm{H}$ such that $F:=\bigcap_{i=1}^{\infty} F i x T_{i} \bigcap F i x(\varphi) \neq \varnothing$ and $T_{i}(\operatorname{Fix} \varphi) \subset F i x(\varphi)$, for all $i \in N$. Let $x_{0} \in H$, $0<\gamma<\frac{\bar{\gamma}}{\alpha}$ and let $\left\{x_{n}\right\}$ be generated by the iterative algorithm (1.3), where $W_{n}$ is a sequence defined by (1.1), and $\left\{\alpha_{n}\right\},\left\{\beta_{n}\right\}$ are sequences in $[0,1]$ satisfying conditions (C1), (C2), (C3).Then $\left\{x_{n}\right\}$ converges strongly $x^{*} \in F$, which also uniquely solves the variational inequality (1.2). 
Proof. We shall divide the proof into several steps.

Step1. The sequence $\left\{x_{n}\right\}$ is bounded.

Since A is strongly positive operator on $\mathrm{H}$, then

$\|A\|=\sup \{|\langle A x, x\rangle|: x \in H\|x\|=1\}$.

$\left.\|\left(1-\beta_{n}\right) I-\alpha_{n} A\right) \|=\sup \left\{1-\beta_{n}-\alpha_{n}\langle A x, x\rangle: x \in H\|x\|=1\right\} \leq 1-\beta_{n}-\alpha_{n} \bar{\gamma}$.

Let $p \in F$, by lemma 2.2 in [1], [10], we have

$$
\begin{aligned}
\left\|x_{n+1}-p\right\| & =\left\|\alpha_{n}\left(\gamma f\left(x_{n}\right)-A p\right)+\beta_{n}\left(x_{n}-p\right)+\left(\left(1-\beta_{n}\right) I-\alpha_{n} A\right)\left(T_{\mu_{n}} W_{n} x_{n}-p\right)\right\| \\
& \leq \alpha_{n}\left\|\gamma f\left(x_{n}\right)-\gamma f(p)\right\|+\alpha_{n}\|\gamma f(p)-A p\|+\beta_{n}\left\|x_{n}-p\right\| \\
& +\left(1-\beta_{n}-\alpha_{n} \bar{\gamma}\right)\left\|T_{\mu_{n}} W_{n} x_{n}-p\right\| \leq\left(1-(\bar{\gamma}-\gamma \alpha) \alpha_{n}\right)\left\|x_{n}-p\right\|+\alpha_{n} \mid \gamma f(p)-A p \|
\end{aligned}
$$

It follows from induction that

$$
\left\|x_{n}-p\right\| \leq \max \left\{\left\|x_{0}-p\right\|, \frac{\|\gamma f(p)-A p\|}{\bar{\gamma}-\gamma \alpha}\right\}=M
$$

Step 2. $\lim _{n \rightarrow \infty}\left\|x_{n}-T_{\mu_{n}} w_{n} x_{n}\right\|=0$.

$\left\|x_{n}-T_{\mu_{n}} w_{n} x_{n}\right\| \leq\left\|x_{n}-x_{n+1}\right\|+\left\|x_{n+1}-T_{\mu_{n}} w_{n} x_{n}\right\|$ we have:

$\left\|x_{n+1}-T_{\mu_{n}} w_{n} x_{n}\right\| \leq \alpha_{n}\left\|\gamma f\left(x_{n}\right)-A T_{\mu_{n}} w_{n} x_{n}\right\|+\beta_{n}\left\|x_{n}-T_{\mu_{n}} w_{n} x_{n}\right\|$ so we have

$\left\|x_{n}-T_{\mu_{n}} w_{n} x_{n}\right\| \leq \frac{1}{1-\beta_{n}}\left[\alpha_{n}\left\|\gamma f\left(x_{n}\right)-A T_{\mu_{n}} w_{n} x_{n}\right\|+\left\|x_{n}-x_{n+1}\right\|\right]$

Since $W_{n}, T_{\mu_{n}}$ are nonexpansive, we have

$\alpha_{n}\left\|\gamma f\left(x_{n}\right)-A T_{\mu_{n}} w_{n} x_{n}\right\| \leq \alpha_{n}\left((\gamma \alpha+\|A\|) M_{0}+\|\gamma f(p)-A p\|\right)$ take

$\left.L_{0}=(\gamma \alpha+\|A\|) M_{0}+\|\gamma f(p)-A p\|\right)$ since $\lim _{n \rightarrow \infty} \alpha_{n}=0$, there exists $K_{0} \in N$ such that $\alpha_{n}<\frac{\delta}{L_{0}}$,

for all $n>K_{0}$ therefore we have

$$
\left\|x_{n}-T_{\mu_{n}} w_{n} x_{n}\right\| \leq \frac{1}{1-\beta_{n}}\left[\delta+\left\|x_{n}-x_{n+1}\right\|\right] \quad \forall n>K_{0}
$$

It sufficient to show that $\lim _{n \rightarrow \infty}\left\|x_{n}-x_{n+1}\right\|=0$

Define

$$
x_{n+1}=\left(1-\beta_{n}\right) y_{n}+\beta_{n} x_{n}, \quad n \geq 0
$$

Observe that from the definition of $y_{n}$, we obtain

$$
\begin{aligned}
y_{n+1}-y_{n}= & \frac{x_{n+2}-\beta_{n+1} x_{n+1}}{1-\beta_{n+1}}-\frac{x_{n+1}-\beta_{n} x_{n}}{1-\beta_{n}}, \\
= & \frac{\alpha_{n+1}}{1-\beta_{n+1}}\left[\gamma f\left(x_{n+1}\right)-A T_{\mu_{n+1}} W_{n+1} x_{n+1}\right]-\frac{\alpha_{n}}{1-\beta_{n}}\left[\gamma f\left(x_{n}\right)-A T_{\mu_{n}} W_{n} x_{n}\right] \\
& +T_{\mu_{n+1}} W_{n+1} x_{n+1}-T_{\mu_{n}} W_{n} x_{n}
\end{aligned}
$$

This implies that 
$\left\|y_{n+1}-y_{n}\right\|$

$\left.\leq\left[\frac{\alpha_{n+1}}{1-\beta_{n+1}}\left\|\gamma f\left(x_{n+1}\right)\right\|+\left\|A T_{\mu_{n+1}} W_{n+1} x_{n+1}\right\|\right]-\frac{\alpha_{n}}{1-\beta_{n}}\left\|\gamma f\left(x_{n}\right)\right\|+\left\|A T_{\mu_{n}} W_{n} x_{n}\right\|\right]+$

$\left\|T_{\mu_{n+1}} W_{n+1} x_{n+1}-T_{\mu_{n}} W_{n} x_{n}\right\|$

We have

$\left\|T_{\mu_{n+1}} W_{n+1} x_{n+1}-T_{\mu_{n}} W_{n} x_{n}\right\|$

$\leq\left\|T_{\mu_{n+1}} W_{n+1} x_{n+1}-T_{\mu_{n+1}} W_{n} x_{n}\right\|+\left\|T_{\mu_{n+1}} W_{n} x_{n}-T_{\mu_{n}} W_{n} x_{n}\right\|$

$\leq\left\|W_{n+1} x_{n+1}-W_{n} x_{n}\right\|+\left\|T_{\mu_{n+1}} W_{n} x_{n}-T_{\mu_{n}} W_{n} x_{n}\right\|$

$\leq\left\|W_{n+1} x_{n+1}-W_{n+1} x_{n}\right\|+\left\|W_{n+1} x_{n}-W_{n} x_{n}\right\|+\left\|T_{\mu_{n+1}} W_{n} x_{n}-T_{\mu_{n}} W_{n} x_{n}\right\|$

$\leq\left\|x_{n+1}-x_{n}\right\|+\left\|W_{n+1} x_{n}-W_{n} x_{n}\right\|+\left\|T_{\mu_{n+1}} W_{n} x_{n}-T_{\mu_{n}} W_{n} x_{n}\right\|$

Since $T_{i}$ and $U_{n, i}$ are nonexpansive, from (1.1), we have

$\left\|W_{n+1} x_{n}-W_{n} x_{n}\right\|=\left\|\lambda_{1} T_{1} U_{n+1,2} x_{n}-\lambda_{1} T_{1} U_{n, 2} x_{n}\right\| \leq \lambda_{1}\left\|U_{n+1,2} x_{n}-U_{n, 2} x_{n}\right\| \ldots \ldots \leq M_{1} \prod_{i=1}^{n} \lambda_{i}$

Where $M_{1} \geq 0$ is an appropriate constant such that $\left\|U_{n+1, n+1} x_{n}-U_{n, n+1} x_{n}\right\| \leq M_{1}$ using (2.3) and (2.4) we have $\left\|T_{\mu_{n+1}} W_{n+1} x_{n+1}-T_{\mu_{n}} W_{n} x_{n}\right\|$

$$
\leq\left\|x_{n+1}-x_{n}\right\|+M_{1} \prod_{i=1}^{n} \lambda_{i}+\left\|T_{\mu_{n+1}} W_{n} x_{n}-T_{\mu_{n}} W_{n} x_{n}\right\|
$$

Substituting (2.5) in (2.2), we have

$\left\|y_{n+1}-y_{n}\right\|-\left\|x_{n+1}-x_{n}\right\| \leq$

$\left.\left.\frac{\alpha_{n+1}}{1-\beta_{n+1}}\left\|\gamma f\left(x_{n+1}\right)\right\|+\left\|A T_{\mu_{n+1}} W_{n+1} x_{n+1}\right\|\right]-\frac{\alpha_{n}}{1-\beta_{n}}\left\|\gamma f\left(x_{n}\right)\right\|+\left\|A T_{\mu_{n}} W_{n} x_{n}\right\|\right]+$

$M_{1} \prod_{i=1}^{n} \lambda_{i}+\left\|T_{\mu_{n+1}} W_{n} x_{n}-T_{\mu_{n}} W_{n} x_{n}\right\|$

Which implies that (noting that $(\mathrm{C} 1)$ and $0<\lambda_{i} \leq b<1$, for $i \geq 1$ )

$$
\limsup _{n \rightarrow \infty}\left\|y_{n+1}-y_{n}\right\|-\left\|x_{n+1}-x_{n}\right\| \leq 0
$$

Hence by Lemma 2.5, we have $\lim _{n \rightarrow \infty}\left\|y_{n}-x_{n}\right\|=0$. Consequently

$\lim _{n \rightarrow \infty}\left\|x_{n+1}-x_{n}\right\|=\lim _{n \rightarrow \infty}\left(1-\beta_{n}\right)\left\|y_{n}-x_{n}\right\|=0$. This together with (2.1) implies that

$\lim _{n \rightarrow \infty}\left\|x_{n}-T_{\mu_{n}} w_{n} x_{n}\right\|=0$.

Step 3. $\limsup _{n \rightarrow \infty} \sup _{y \in D} T_{\mu_{n}} y-T_{t} T_{\mu_{n}} y \|=0, \quad \forall t \in S$

Set $D=\left\{y \in H:\|y-p\| \leq M_{0}\right\}$. We point out that $\mathrm{D}$ is a bounded closed convex set, $\left\{x_{n}\right\} \subset D$ being invariant under $\varphi$ and $W_{n}$, for all $n \in N$

Let $\varepsilon>0$. By Theorem 1.2 in [6], there exists $\delta>0$ such that 


$$
\overline{c o} F_{\delta}\left(T_{t} ; D\right)+B_{\delta} \subset F_{\varepsilon}\left(T_{t} ; D\right), \quad \forall t \in S .
$$

Also by Corollary 1.1 in [6], there exists a natural number $\mathrm{N}$ such that

$\left\|\frac{1}{N+1} \sum_{i=0}^{N} T_{t^{i} s} y-T_{t}\left(\frac{1}{N+1} \sum_{i=0}^{N} T_{t^{i} s} y\right)\right\| \leq \delta, \forall t, s \in S, y \in D$

Let $t \in S$. Since $\left\{\mu_{n}\right\}$ is strongly left regular, there exists $N_{0} \in \mathrm{N}$ such that $\left\|\mu_{n}-l_{t^{i}}^{*} \mu_{n}\right\| \leq \frac{\delta}{\left(M_{0}+\|x\|\right)}, \quad \forall n \geq N_{0}, i=1,2, \ldots, N$.

Then we have

$$
\begin{aligned}
& \sup _{y \in D} T_{\mu_{n}} y-\int \frac{1}{N+1} \sum_{i=0}^{N} T_{t^{i} s} y d \mu_{n}(s) \|=\operatorname{supsup}_{y \in D\|\nexists\|=1}\left|\left\langle T_{\mu_{n}} y, z\right\rangle-\left\langle\int \frac{1}{N+1} \sum_{i=0}^{N} T_{t^{i} s} y d \mu_{n}(s), z\right\rangle\right| \\
& =\operatorname{supsup}_{y \in D\|\nexists\|=1}\left|\frac{1}{N+1} \sum_{i=0}^{N}\left(\mu_{n}\right)_{s}-\frac{1}{N+1} \sum_{i=0}^{N}\left(\mu_{n}\right)_{s}\left\langle T_{t^{i} s} y, z\right\rangle\right| \\
& \leq \frac{1}{N+1} \sum_{i=0}^{N} \sup _{y \in D\|\| \|=1}\left(\mu_{n}\right)_{s}\left\langle T_{t^{i} s} y, z\right\rangle-\left(l_{t^{i}}^{*} \mu_{n}\right)_{s}\left\langle T_{s} y, z\right\rangle \mid \\
& \leq \max _{i=1,2, \ldots, N} \| \mu_{n}-l_{t^{i}}^{*} \mu_{n}\left(M_{0}+\|p\|\right) \leq \delta, \quad \forall n \geq N_{0} .
\end{aligned}
$$

By Lemma 2.2, we have

$$
\int \frac{1}{N+1} \sum_{i=0}^{N} T_{t^{i} s} y d \mu_{n}(s) \in \overline{c o}\left\{\int \frac{1}{N+1} \sum_{i=0}^{N} T_{t^{i}}\left(T_{s} y\right): s \in S\right\} \text {. }
$$

It follows from 2.6, 2.7, 2.8 and 2.9 that

$T_{\mu_{n}} y \in \overline{c o}\left\{\frac{1}{N+1} \sum_{i=0}^{N} T_{t^{i} s} y: s \in S\right\}+B_{\delta} \subset \overline{c o} F_{\delta}\left(T_{t} ; D\right)+B_{\delta} \subset F_{\mathrm{o}}\left(T_{t} ; D\right) \quad \forall y \in D, n \geq N_{0}$.

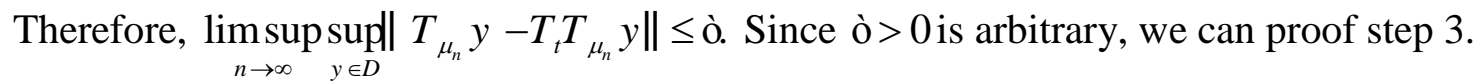

Step 4. $\lim _{n \rightarrow \infty}\left\|x_{n}-T_{t} x_{n}\right\|=0, \forall t \in S$.

We have shown in Step 2 that $\alpha_{n}\left\|\gamma f\left(x_{n}\right)-A T_{\mu_{n}} w_{n} x_{n}\right\| \leq \alpha_{n}\left((\gamma \alpha+\|A\|) M_{0}+\|\gamma f(p)-A p\|\right) \leq \frac{\delta}{L_{0}} L_{0}=\delta$ for all $n>K_{0}$ Using Step 2 we can assume, there exists $K_{1} \in N$ such that for all $n \geq K_{1}$,

$$
\beta_{n}\left(x_{n}-T_{\mu_{n}} w_{n} x_{n}\right) \in B_{\frac{\delta}{2}}
$$

From Lemma 2.7 we have $\left\|W_{n} x_{n}-p\right\| \# W_{n} x_{n}-W_{n} p\left\|\sharp x_{n}-p\right\| \leq M_{0}$, from definition $\mathrm{D}$ we have $W_{n} x_{n} \in D$. Therefore by Step 3 we have $T_{\mu_{n}} W_{n} x_{n} \in F_{\delta}\left(T_{t} ; D\right)$, so we have

$$
\begin{aligned}
x_{n+1} & =\alpha_{n}\left[\gamma f\left(x_{n}\right)-A T_{\mu_{n}} W_{n} x_{n}\right]+\beta_{n}\left[x_{n}-T_{\mu_{n}} W_{n} x_{n}\right]+T_{\mu_{n}} W_{n} x_{n} \\
& \in B_{\frac{\delta}{2}}+B_{\frac{\delta}{2}}+F_{\delta}\left(T_{t} ; D\right) \subset B_{\delta}+F_{\delta}\left(T_{t} ; D\right) \subset F_{\mathrm{o}}\left(T_{t} ; D\right)
\end{aligned}
$$


For all $n \geq K_{2}$ in which $K_{2}=\max \left\{K_{0}, K_{1}\right\}$, so we have:

$$
\left\|x_{n}-T_{t} x_{n}\right\|<\mathrm{o}
$$

Step 5. There exists a unique $x^{*} \in F$ such that

$$
\limsup _{n \rightarrow \infty}\left\langle x_{n}-x^{*}, \gamma f\left(x^{*}\right)-A x^{*}\right\rangle \leq 0
$$

$P_{F}(I-A+\gamma f)$ is a contraction of $\mathrm{H}$ into itself because

$\left\|P_{F}(I-A+\gamma f) x-P_{F}(I-A+\gamma f) y\right\| \leq\|(I-A+\gamma f) x-(I-A+\gamma f) y\|$

$\leq\|I-A\|\|x-y\|+\gamma d\|x-y\| \leq(1-(\bar{\gamma}-\gamma \alpha))\|x-y\|$,

By Banach contraction principal, $P_{F}(I-A+\gamma f)$ has a unique fixed point $x^{*} \in F$. Then by

Lemma 2.4, we have

$$
\left\langle\gamma f\left(x^{*}\right)-A x^{*}, y-x^{*}\right\rangle \leq 0 \quad \forall y \in F
$$

We take a subsequence $\left\{x_{n_{k}}\right\}$ of $\left\{x_{n}\right\}$ such that

$$
\limsup _{n \rightarrow \infty}\left\langle x_{n}-x^{*}, \gamma f\left(x^{*}\right)-A x^{*}\right\rangle=\lim _{k \rightarrow \infty}\left\langle x_{n_{k}}-x^{*}, \gamma f\left(x^{*}\right)-A x^{*}\right\rangle
$$

We may also assume that $x_{n_{k}} \square z$, using Step 4 and Lemma 2.3 we have $z \in$ Fix $(\varphi)$. We will show that $z \in F$, from Lemma 2.7 it follows that $\bigcap_{i=1}^{\infty} F i x T_{i}=F i x(W)$. So we must show that $z \in$ Fix $(W)$. Assume that $W z \neq z$, since $z \in$ Fix $(\varphi)$, and by assumption, $T_{i}$ Fix $\varphi \subset$ Fix $\varphi$

We have $T_{i} z \in$ Fix $\varphi \quad \forall i \in N$, so $W_{n} z \in$ Fix $\varphi$. Hence $T_{\mu_{n}} W_{n} z=W_{n} z$ using Opial property of Hilbert space, since $x_{n_{k}} \square z$, we have

$$
\liminf _{k \rightarrow \infty} x_{n_{k}}-x\left\|>\liminf _{k \rightarrow \infty}\right\| x_{n_{k}}-z \| \quad \forall x \neq z
$$

Since $W z \neq z$ by using Step 2 and Lemma2.8, we have $\liminf _{k \rightarrow \infty} \mid x_{n_{k}}-z\left\|<\liminf _{k \rightarrow \infty}\right\| x_{n_{k}}-W z \|$

$$
\begin{aligned}
& \left.\leq \liminf _{k \rightarrow \infty}\left\|x_{n_{k}}-T_{\mu_{n_{k}}} W_{n_{k}} x_{n_{k}}\right\|+\left\|T_{\mu_{n_{k}}} W_{n_{k}} x_{n_{k}}-T_{\mu_{n_{k}}} W_{n_{k}} z\right\|+\left\|T_{\mu_{n_{k}}} W_{n_{k}} z-W z\right\|\right] \\
& \left.\leq \liminf _{k \rightarrow \infty}\left\|x_{n_{k}}-T_{\mu_{n_{k}}} W_{n_{k}} x_{n_{k}}\right\|+\left\|x_{n_{k}}-z\right\|+\left\|W_{n_{k}} z-W z\right\|\right] \\
& =\liminf _{k \rightarrow \infty}\left\|x_{n_{k}}-z\right\|
\end{aligned}
$$

This is a contradiction. Therefore $z \in F$. Noticing 2.10, 2.11 and $x_{n_{k}} \square z$ we have:

$$
\limsup _{n \rightarrow \infty}\left\langle x_{n}-x^{*}, \gamma f\left(x^{*}\right)-A x^{*}\right\rangle=\left\langle z-x^{*}, \gamma f\left(x^{*}\right)-A x^{*}\right\rangle \leq 0 \text {. }
$$

Step6. The sequence $\left\{x_{n}\right\}$ converges strongly to $x^{*}$. By Lemma 2.6 we have

$$
\begin{aligned}
\left\|x_{n+1}-x^{*}\right\|^{2} & =\left\|\alpha_{n}\left(\gamma f\left(x_{n}\right)-A x^{*}\right)+\beta_{n}\left(x_{n}-x^{*}\right)+\left(\left(1-\beta_{n}\right) I-\alpha_{n} A\right)\left(T_{\mu_{n}} W_{n} x_{n}-x^{*}\right)\right\|^{2} \\
& \left.\leq\left[\beta_{n}\left\|x_{n}-x\right\|+\left(1-\beta_{n}\right)-\alpha_{n} \bar{\gamma}\right)\left\|x_{n}-x^{*}\right\|\right]^{2}+2 \alpha_{n}\left\langle\gamma f\left(x_{n}\right)-A x^{*}, x_{n+1}-x^{*}\right\rangle \\
& =\left(1-\alpha_{n} \bar{\gamma}\right)^{2}\left\|x_{n}-x^{*}\right\|^{2}+2 \alpha_{n}\left\langle\gamma f\left(x_{n}\right)-A x^{*}, x_{n+1}-x^{*}\right\rangle
\end{aligned}
$$

On the other hands, 


$$
\begin{aligned}
& \left\langle\gamma f\left(x_{n}\right)-\gamma f\left(x^{*}\right), x_{n+1}-x^{*}\right\rangle \leq \gamma \alpha\left\|x_{n}-x^{*}\right\|\left\|x_{n+1}-x^{*}\right\| \\
& \leq \gamma \alpha\left\|x_{n}-x^{*}\right\| \sqrt{\left(1-\alpha_{n} \bar{\gamma}\right)^{2}\left\|x_{n}-x^{*}\right\|^{2}+2 \alpha_{n}\left\langle\gamma f\left(x_{n}\right)-A x^{*}, x_{n+1}-x^{*}\right\rangle} \\
& \quad \leq \gamma \alpha\left(1-\alpha_{n} \bar{\gamma}\left\|x_{n}-x^{*}\right\|^{2}+\gamma d\left\|x_{n}-x\right\| \sqrt{2\left\langle\gamma f\left(x_{n}\right)-A x^{*}, x_{n+1}-x^{*}\right\rangle} \sqrt{\alpha_{n}}\right.
\end{aligned}
$$

Since $\left\{x_{n}\right\}$ is bounded, we can take a constant $G_{0}>0$ such that

$$
\gamma d\left\|x_{n}-x^{*}\right\| \sqrt{2\left\langle\gamma f\left(x_{n}\right)-A x^{*}, x_{n+1}-x^{*}\right\rangle} \sqrt{\alpha_{n}}<G_{0}, \forall n \in N
$$

So from the above and 2.12 we reach the following

$\mid x_{n+1}-x \|^{2}$

$\leq\left(1-\alpha_{n} \bar{\gamma}\right)^{2}\left\|x_{n}-x^{*}\right\|^{2}+2 \alpha_{n}\left\langle\gamma f\left(x_{n}\right)-\gamma f\left(x^{*}\right), x_{n+1}-x^{*}\right\rangle+2 \alpha_{n}\left\langle\gamma f\left(x^{*}\right)-A x^{*}, x_{n+1}-x^{*}\right\rangle$

$\leq\left(1-\alpha_{n} \bar{\gamma}\right)^{2}\left\|x_{n}-x\right\|^{2}+2 \alpha_{n} \gamma \alpha\left(1-\alpha_{n} \bar{\gamma}\left\|x_{n}-x^{*}\right\|^{2}+2 \alpha_{n} G_{0} \sqrt{\alpha_{n}}\right.$

$+2 \alpha_{n}\left\langle\gamma f\left(x^{*}\right)-A x^{*}, x_{n+1}-x^{*}\right\rangle$

$\left.=\left(1-\left(2(\bar{\gamma}-\gamma \alpha)+\alpha_{n} \bar{\gamma}(2 \gamma \alpha-\bar{\gamma})\right) \alpha_{n}\right)\right)\left\|x_{n}-x^{*}\right\|^{2}+2 \alpha_{n} G_{0} \sqrt{\alpha_{n}}+2 \alpha_{n}\left\langle\gamma f\left(x^{*}\right)-A x^{*}, x_{n+1}-x^{*}\right\rangle$

It then follows that

$$
\left\|x_{n+1}-x^{2}\right\|^{2} \leq\left(1-\gamma_{n}\right)\left\|x_{n}-x\right\|^{2}+\alpha_{n} \delta_{n} .
$$

Where

$$
\begin{aligned}
& \gamma_{n}=\left(2(\bar{\gamma}-\gamma \alpha)+\alpha_{n} \bar{\gamma}(2 \gamma \alpha-\bar{\gamma})\right) \alpha_{n}, \\
& \delta_{n}=2 G_{0} \sqrt{\alpha_{n}}+2\left\langle\gamma f\left(x^{*}\right)-A x^{*}, x_{n+1}-x^{*}\right\rangle
\end{aligned}
$$

By Step 4, we get $\lim \sup \delta_{n} \leq 0$. Since $\alpha_{n} \rightarrow 0$ and $0<\bar{\gamma}-\gamma \alpha$, we may assume, with no loss of generality, that $\alpha_{n}<\|A\|^{-1}$ and

$$
0<2(\bar{\gamma}-\gamma \alpha)+\alpha_{n} \bar{\gamma}(2 \gamma \alpha-\bar{\gamma})<1
$$

This together with applying Lemma 2.9 to 2.13 concludes that $\lim _{n \rightarrow \infty}\left\|x_{n}-x\right\|=0$.

\section{References}

1. A. T. Lau, N. Shioji and W. Takahashi. Existences of nonexpansive retractions for amenable semigroups of nonexpansive mappings and nonlinear ergodic theorems in Banach spaces. J. Funct. Anual. 161(1999), $62-75$

2. G. Marino and H. K. Xu, A general iterative method for nonexpansive mappings in Hilbert spaces, j. Math. Anal. Appl. 318 (2006), no. 1, 43-52.

3. H. K. Xu, Viscosity approximation methods for nonexpansive mappings, J. Math. Anal. Appl. 298 (2004), 279291.

4. H. Piri and H. Vaezi, Strong convergence of a generalized iterative method for semigroups of nonexpansive mappings in Hilbert spaces, Fixed Point Theory Appl. (2010). doi:10. 1155/2010/907275.

5. K. Shimoji and W. Takahashi, Strong convergence to common fxed points of infnite nonexpansive mappings and applications, Taiwanese J. Math. 5 (2001), no. 2, 387404

6. R. E Bruck, On the convex approximation property and the asymptotic behavior of nonlinear contractions in Banach spaces, Israel J. Math. 38(1981), 304-314. 
7. Sh. Banerjee, B. S. Choudhury, Weak and strong convergence theorems of a new iterative process with errors for common fixed points of a finite families of asymptotically nonexpansive mappings in the intermediate sense in Banach spaces. TJMCS, 11(2014), 79-85.

8. S. S. Zhang, J. H. W. Lee, and C. K. Chan, Algorithms of common solutions to quasi variational inclusion and fixed point problems, Appl. Math. Mech. 29(2008), no. 5, 571581.

9. T. Suzuki, Strong convergence of Krasnoselskii and Mann's type sequences for oneparameter nonexpansive semigroups without Bochner integrals, J. Math. Anal. Appl. 305 (2005), no. 1, 227239.

10. W. Takahashi, A nonlinera ergodic theorem for an amenable semigroup of nonexpansive mappings in Hilbert space. Proc. Amer. Math. Soc. 81(1981). 253-256.

11. W, Takahashi, Nonlinear Functional Analysis. Yokohama Publishers, Yokohama, 2000.

12. Y. Yao, A general iterative method for a finite family of nonexpansive mappings, Nonlinear Anal. 66 (2007), 2676-2687. 